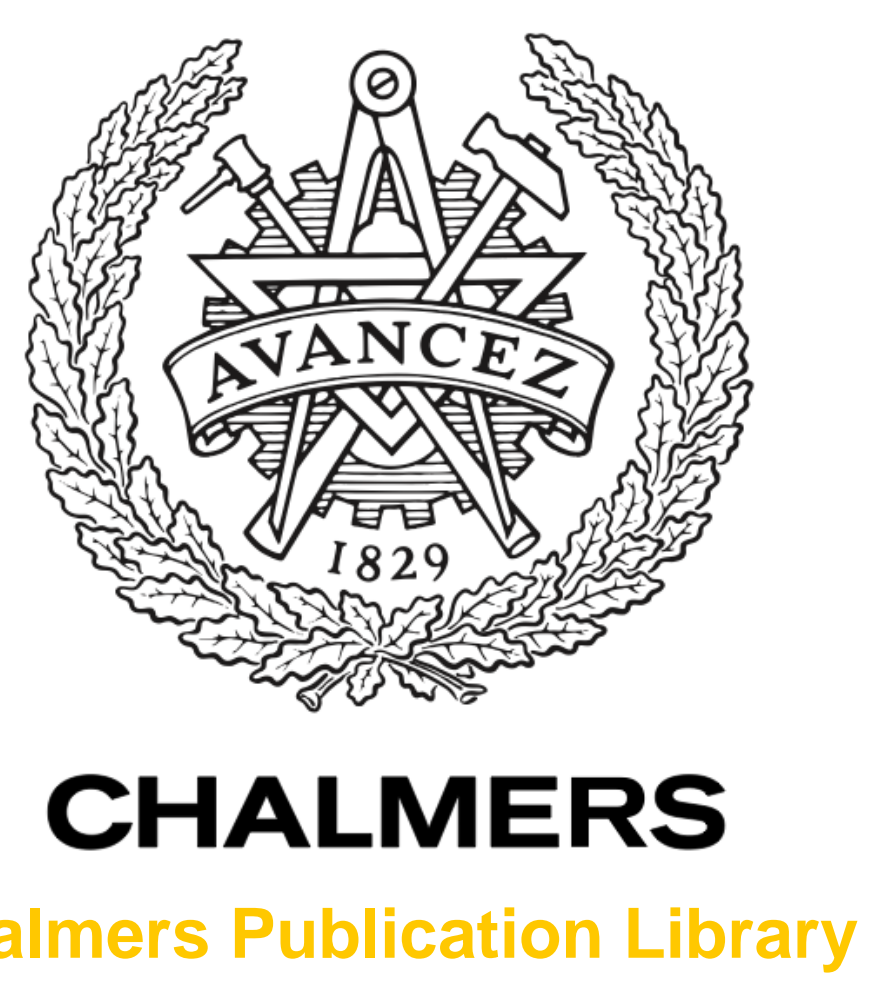

Chalmers Publication Library

\title{
Linearization Study of a Highly Efficient CMOS-GaN RF Pulse Width Modulation Based Transmitter
}

This document has been downloaded from Chalmers Publication Library (CPL). It is the author's version of a work that was accepted for publication in:

\section{European Microwave Conference}

Citation for the published paper:

Özen, M. ; Andersson, C. ; Eriksson, T. (2012) "Linearization Study of a Highly Efficient

CMOS-GaN RF Pulse Width Modulation Based Transmitter". European Microwave

Conference pp. 137-139.

Downloaded from: http://publications.lib.chalmers.se/publication/170026

Notice: Changes introduced as a result of publishing processes such as copy-editing and formatting may not be reflected in this document. For a definitive version of this work, please refer to the published source. Please note that access to the published version might require a subscription. 


\title{
Linearization Study of a Highly Efficient CMOS-GaN RF Pulse Width Modulation Based Transmitter
}

\author{
Mustafa Özen*, Christer M. Andersson*, Thomas Eriksson*, Mustafa Acar ${ }^{\dagger}$, Rik Jos ${ }^{\dagger *}$ \\ and Christian Fager* \\ * Chalmers University of Technology, Gothenburg 412 96, Sweden \\ *mustafa.ozen@chalmers.se \\ $\dagger$ NXP Semiconductors, Eindhoven, 5656AE, The Netherlands \\ ${ }^{\dagger}$ rik.jos@nxp.com
}

\begin{abstract}
This paper studies linearity of a $2 \mathrm{GHz}, 10$ Watt peak output power RF pulse width modulation (RF-PWM) based transmitter. The transmitter incorporates a tunable load network class-E PA as the final output stage. The tunable load network enables dynamic optimization of the class-E along with the duty cycle resulting in high efficiency over a wide range of output power levels. A digital predistiortion based linearization scheme is proposed to enhance the linearity of the transmitter. After linearization, the transmitter exhibits an adjacent channel power ratio (ACPR) of $-45 \mathrm{dBc}$ with a $3.84 \mathrm{MHz}, 6.7 \mathrm{~dB}$ peak-toaverage power ratio (PAPR) W-CDMA signal. The average drain efficiency of the GaN HEMT output stage is $67 \%$ and the total transmitter efficiency is $54 \%$.
\end{abstract}

\section{INTRODUCTION}

A radio frequency pulse width modulation (RF-PWM) based transmitter consists of three main parts: a modulator, a switch mode power amplifier (SMPA) and a band pass postPA filter. The modulator encodes the envelope information of the communication signal to the duty cycle (pulse width) of a carrier frequency square wave. Phase information is presented by the timing of the pulses. The resulting pulse train is then amplified with an highly efficient SMPA. Post PA filtering is necessary to remove the spurious products at the harmonics of the carrier to recover the signal around the carrier frequency [1].

The main issue of RF-PWM transmitter architecture is that conventional SMPAs, e.g. class-D, class-E, provides high efficiency only for a fixed duty cycle. Variable duty cycle signals at the input creates severe switching losses decreasing the average efficiency with modulated signals [2]. However, recently there has been studies on dynamically tunable load network class-E PAs for energy efficient amplification of RFPWM signals [3], [4]. The main principle is to dynamically optimize the load network along with the duty cycle to preserve the class-E switching conditions for high average efficiency. Characterization results of such PAs with continuous wave signals have been presented previously in [3], [5] which have proved the feasibility of this interesting idea to achieve high efficiency over a wide range of back-off levels. However, linearity of such transmitters is yet to be studied.
In this paper, linearity of an RF-PWM transmitter that employs a tunable reactive load impedance class-E PA is studied with realistic communication signals. A digital predistortion based linearization scheme is proposed to enhance the linearity. The results clearly demonstrate the feasibility of the architecture for energy efficient linear amplification of realistic communication signals.

\section{RF-PWM TRANSMITTER}

The block diagram of the transmitter is shown in Fig. 1. In the transmitter, RF-PWM signals are generated with an inverter implemented in a commercial $65 \mathrm{~nm}$ CMOS process [6]. Ideally, gate bias voltages of both NMOS and PMOS transistors in the inverter should be varied to control the duty cycle. However, our experiments have shown that keeping the NMOS voltage fixed does not degrade the performance significantly in terms of efficiency and output power dynamic range. The duty cycle is therefore controlled by only varying the gate bias voltage of the PMOS transistor, $V_{g P}$, in the inverter [7].

The RF-PWM signals are amplified with a $2 \mathrm{GHz}, 10 \mathrm{~W}$ peak output power, GaN HEMT (high electron mobility transistor) (Cree CGH60015D) tunable reactive load impedance class-E PA. The schematic of the PA is shown in Fig. 2. The tunable load network is implemented using in-house (Chalmers University of Technology) high breakdown voltage $\left(V_{B R}>100 \mathrm{~V}\right) \mathrm{SiC}$ varactor diodes [8]. Zero voltage switching (ZVS) condition of the class-E is preserved versus the duty cycle by only varying a single varactor control signal $\left(V_{c}\right)$ thus enabling high efficiency over a wide output power dynamic range.

\section{StATIC CharacterizATION}

For operation of the transmitter, the optimal combination of $V_{c}$ and $V_{g P}$ for the best efficiency has to be identified. This is performed by varying the $V_{c}$ and optimizing the $V_{g P}$ for the best transmitter efficiency based on continuous wave static measurements using a power meter. The efficiency constrained optimal control signals are shown in Fig. 3 and 


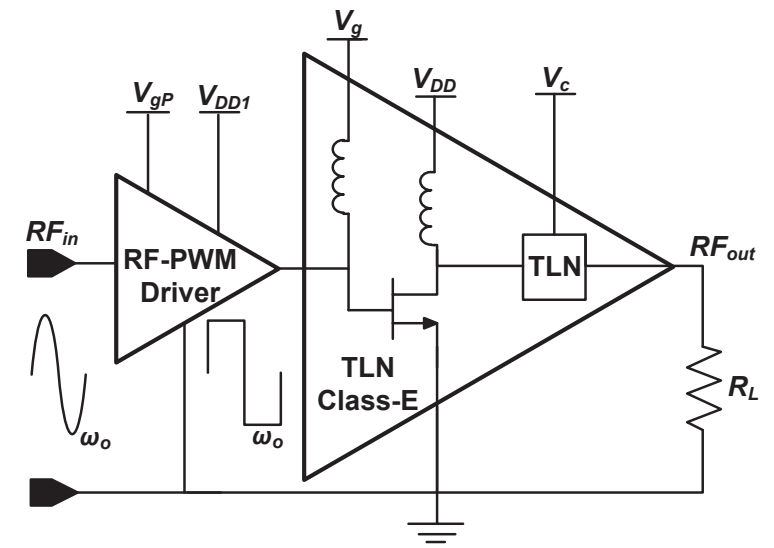

Fig. 1. Block diagram of the RF pulse width modulated class-E transmitter.

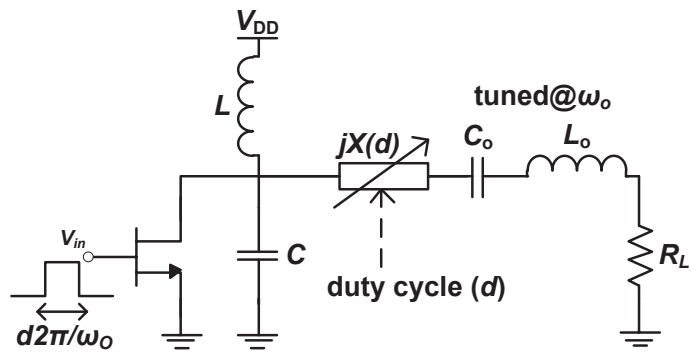

Fig. 2. Class-E PA with tunable reactive load impedance for energy efficient amplification of RF pulse width modulated signals.

the corresponding efficiency performance is shown in Fig. 4. The efficiency definitions follow as

$$
\begin{aligned}
\eta & =\frac{P_{\text {out }}}{P_{D C 1}} \\
\eta_{\text {tot }} & =\frac{P_{\text {out }}}{P_{D C 1}+P_{D C 2}+P_{A}}
\end{aligned}
$$

where $P_{D C 1}$ and $P_{D C 2}$ are the DC powers drawn by the Class-E stage and the modulator stages, respectively. $P_{A}$ is the available RF power from the RF synthesizer applied to the modulator input.

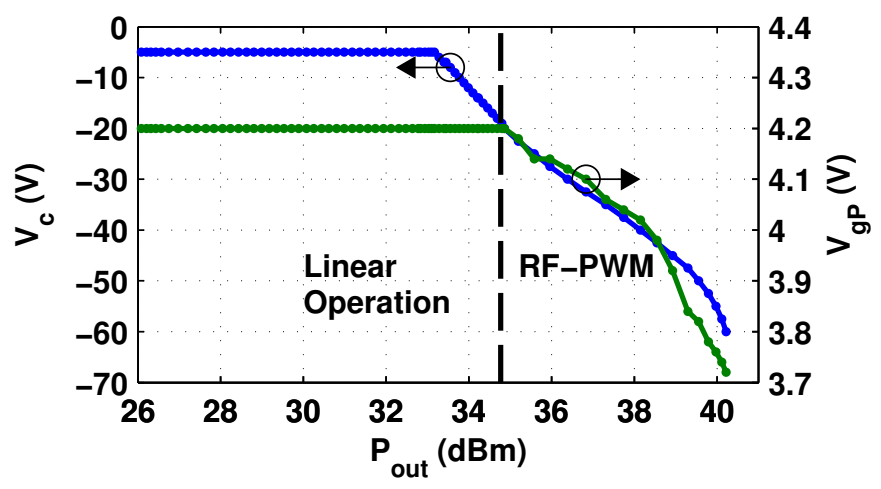

Fig. 3. The optimal varactor and duty cycle control voltages, $V_{c}$ and $V_{g P}$, respectively, versus the output power, $P_{\text {out }}$, at $2 \mathrm{GHz}$ carrier frequency.

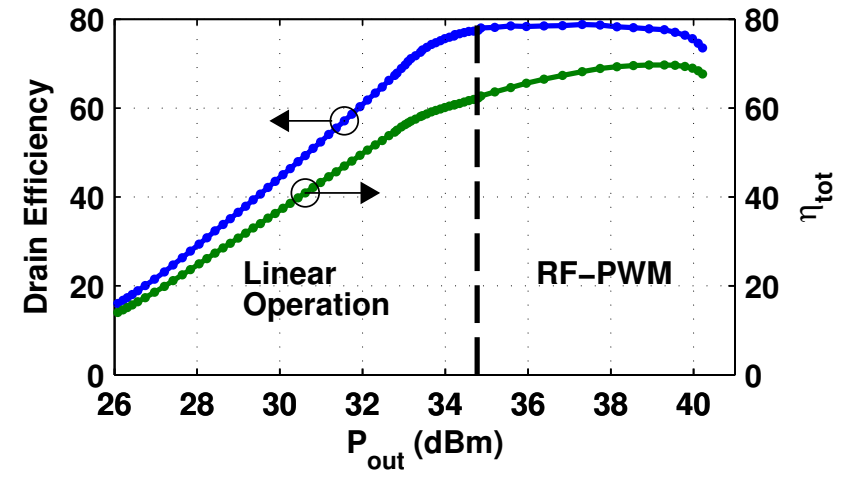

Fig. 4. The measured drain efficiency of the Class-E stage, $\eta$, and the overall transmitter efficiency, $\eta_{\text {tot }}$, versus the output power, $P_{\text {out }}$, at $2 \mathrm{GHz}$ carrier frequency.

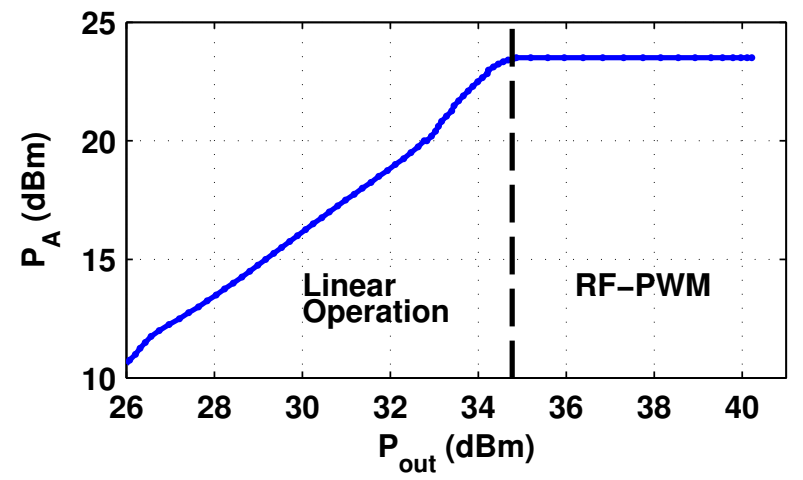

Fig. 5. The available RF input power, $P_{A}$, versus the delivered output power, $P_{\text {out }}$. The carrier frequency is $2 \mathrm{GHz}$.

The output power dynamic range (DR) achieved with RFPWM operation is around $5.5 \mathrm{~dB}$ as seen from Fig. 3. This DR is clearly not enough for linear amplification of modern communication signals, e.g. W-CDMA or LTE. This limitation can, however, be circumvented by operating the transmitter in the linear mode where the output power is directly controlled by the RF input power rather than the duty cycle and varactor voltage. However, as switch mode operation requires CMOS and GaN HEMT devices to be deeply saturated, severe gain expansion is unavoidable in the transition to the linear operation region. This causes large discontinuity in the inputoutput power relationship. The varactor signal can, however, be optimized to obtain a smooth transition between linear and switch modes. The resulting input-output power relation with optimized varactor signal for a smooth transition is shown in Fig. 5.

\section{DyNAMIC ChARACTERIZATION AND LINEARIZATION}

This section covers the dynamic characterization and linearization of the RF-PWM transmitter. First, the measurement setup is introduced. 


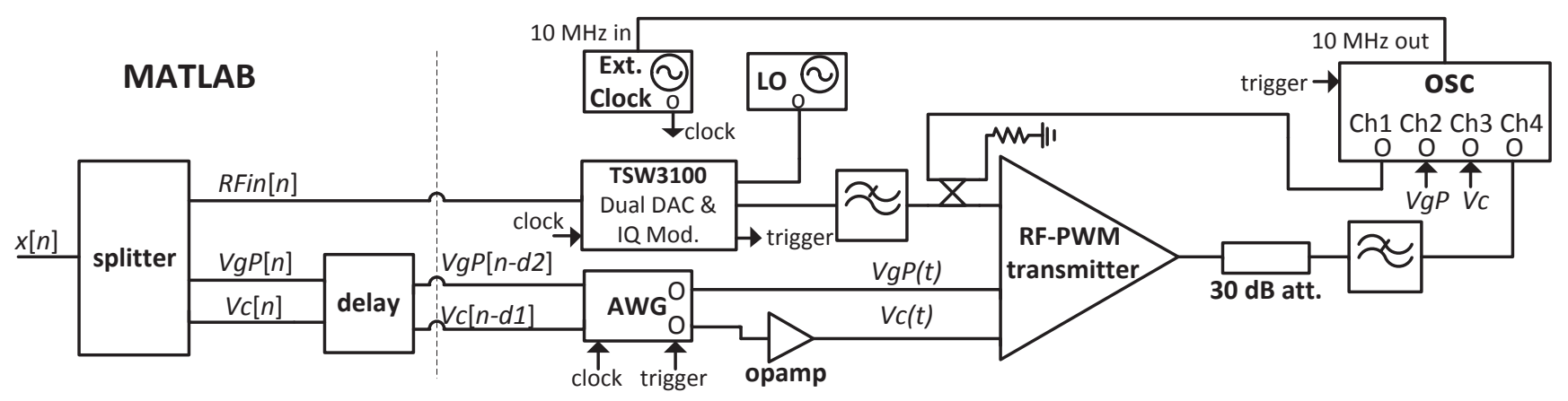

Fig. 6. Block diagram of the measurement setup.

\section{A. Measurement Setup}

A two channel arbitrary waveform generator (Tabor Electronics WW2572A) (AWG) is used to generate the baseband signals. An high speed op-amp (MS Kennedy 600r) based feedback amplifier is used to amplify the varactor control signal to the required level. The duty cycle control signal $V_{g P}$ can directly be generated with the AWG. RF input signal is generated using an evaluation board from Texas Instruments (TSW3100), consisting of two digital to analog converters (DAC) for I and Q branches and an IQ modulator.

For multiple input transmitters, accurate time alignment of the input signals is very crucial for the linearity [9]. Output signal and also the input signals are measured with a sampling oscilloscope. Delay mismatch between the RF signal and the baseband control signals is determined by cross correlating the baseband signals with the RF signal. The mismatch is compensated in the digital domain in MATLAB environment.

It is important to mention that using independent sample clocks even derived from a common $10 \mathrm{MHz}$ reference signal may cause significant delay uncertainty between different signal paths due to phase jitters of the sample clocks. Both TSW3100 and AWG are therefore fed with the same external sample clock for best synchronization and linearization performance. A block diagram of the measurement setup is shown in Fig. 6.

\section{B. Digital Pre-distortion Linearization}

Digital pre-distortion (DPD) scheme used for linearization is shown in Fig. 7. The transmitter is considered as a single input single output system. Efficiency optimized input signals, $V_{c}, V_{g P}$ and $R F_{i n}$ are derived from the predistorted input signal $(\hat{x})$ using a digital splitter constructed from the static CW measurement data. DPD model parameters are identified by comparing the measured output sample $(y)$ and the input sample $(x)$.

A vector switched generalized memory polynomial (VSGMP) model is used as the behavioral model for DPD operation [10]. The VS-GMP quantize the signal according to the signal amplitude in to a number of regions and model each region separately. That model is therefore very suitable for behavioral modeling of the transmitter which has two distinct operating regions, linear and RF-PWM, see Fig. 5.

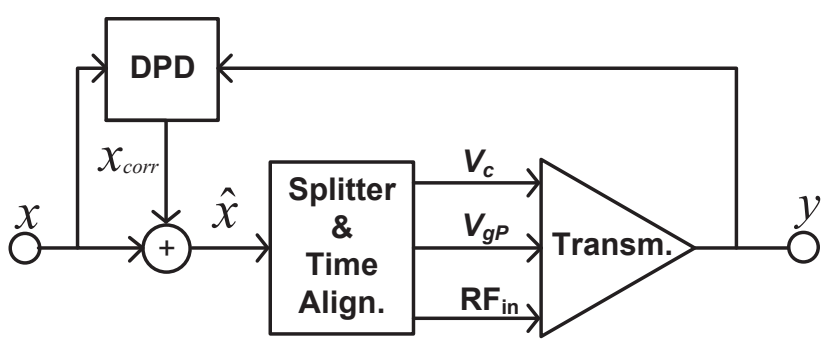

Fig. 7. Digital pre-distortion linearization scheme.

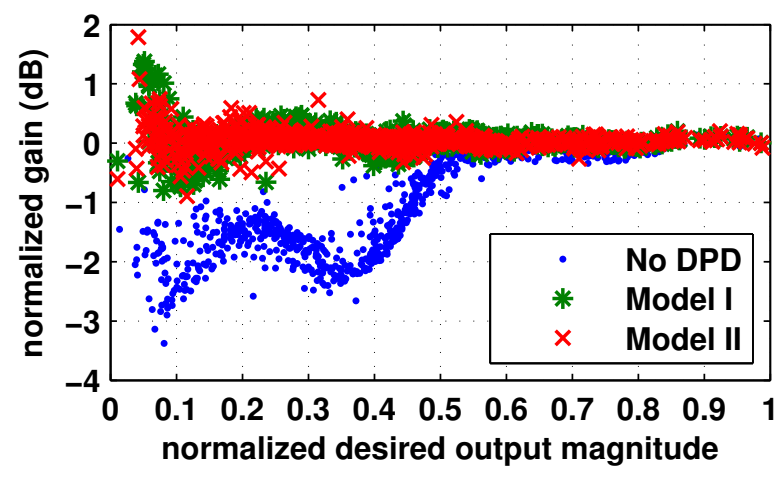

Fig. 8. AM-AM response of the transmitter. Model I and II refer to GMP and vector switched GMP models, respectively.

A $3.84 \mathrm{MHz}$ 6.7 dB PAPR W-CDMA signal is used for the measurements presented through the paper. The AMAM and AM-PM curves are shown in Fig. 8 and Fig. 9, respectively, before and after DPD linearization. High AM-PM distortion for high output amplitudes originates from tuning of the imaginary load impedance versus the duty cycle. This can however be corrected by DPD as observed from the AM-PM curve after linearization.

It can easily be seen from the AM-AM curve that, as expected, the transmitter has distinctly different behaviors for RF-PWM and linear regions. Also, high order of nonlinearity can be observed for the linear operation region, mainly caused by the response of the CMOS driver. A high number of regions for vector quantization is therefore necessary to achieve a good 
TABLE I

SUMMARY OF THE LINEARIZATION RESULTS

\begin{tabular}{c|c|c|c|c|c|c|c|c}
\hline DPD type & Num. of reg. & $(P, M, r)$ & FLOPs & NMSE $(\mathrm{dB})$ & ACPR $(\mathrm{dBc})$ & $P_{\text {out }- \text { avg }}($ Watt $)$ & $\eta_{\text {avg }}$ & $\eta_{\text {tot-avg }}$ \\
\hline- & - & - & - & -12 & -23 & 2.24 & $67 \%$ & $54 \%$ \\
\hline GMP & 1 & $(7,3,1)$ & 672 & -32 & -41 & 2.13 & $65.5 \%$ & $53 \%$ \\
\hline VS GMP & 16 & $(3,3,1)$ & 308 & -35 & -45 & 2.18 & $67 \%$ & $54 \%$ \\
\hline
\end{tabular}

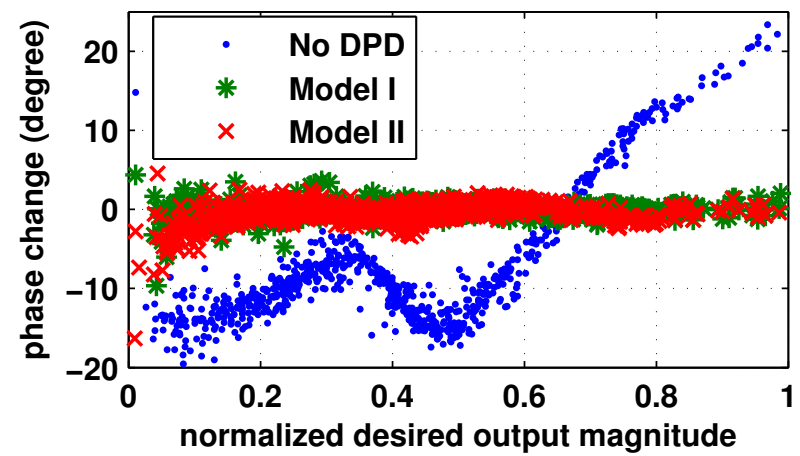

Fig. 9. AM-PM response of the transmitter. Model I and II refer to GMP and vector switched GMP models, respectively.

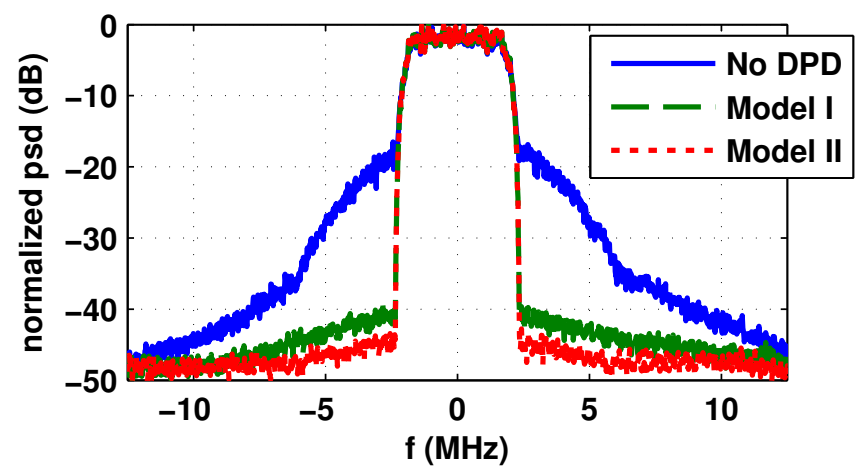

Fig. 10. Normalized power spectral density of the output signal. Model I and II refer to GMP and vector switched GMP models, respectively.

accuracy from the model. It is observed that results improve up to 16 number of regions for the DPD.

Spectrum of the output signal is shown in Fig. 10. By using the VS-GMP based DPD, the adjacent channel power ratio (ACPR) is improved more than $22 \mathrm{~dB}$, which is also 4 $\mathrm{dB}$ better than if a conventional GMP model is used. The linearization results are summarized in Table I. Run-time computational complexity of VS-GMP and GMP models are also compared in the table where number of floating point operations per sample (FLOPs) used as the metric [10]. Also in terms of complexity, the results clearly demonstrate that the VS-GMP is very powerful. Formulas for calculation of number of FLOPs can be found in [10].

\section{Conclusion}

Linearity characteristics of a high efficiency RF-PWM based transmitter is studied. The dynamic range achieved with RFPWM operation is limited by the minimum duty cycle of the modulator. This problem is circumvented by switching to linear mode of operation, i.e. controlling the output amplitude by varying the input amplitude rather than varying the duty cycle. The transmitter thus exhibits two distinct behaviors according to the output amplitude. Vector switched models are therefore most suitable for pre-distortion linearization of the transmitter presented. Average drain efficiency of $67 \%$ and ACPR of $-45 \mathrm{dBc}$ are measured with a $6.7 \mathrm{~dB}$ PAPR WCDMA signal. These results clearly prove the feasibility of the architecture for efficient linear amplification.

\section{ACKNOWLEDGMENT}

This research has been carried out in GigaHertz Centre in a joint project financed by the Swedish Governmental Agency for Innovation Systems (VINNOVA), Chalmers University of Technology, Ericsson AB, Infineon Technologies Austria AG, and NXP Semiconductors BV.

\section{REFERENCES}

[1] F. H. Raab, "Radio frequency pulsewidth modulation," IEEE Transactions on Communications, vol. 21, pp. 958-966, Aug. 1973.

[2] K. Tom, M. Faulkner, and T. Lejon, "Performance analysis of pulse width modulated RF Class-E power amplifier," in IEEE 63rd Vehicular Technology Conference, vol. 4, May 2006, pp. 1807-1811.

[3] M. Özen, R. Jos, C. M. Andersson, M. Acar, and C. Fager, "High efficiency radio frequency pulse width modulation of Class-E power amplifiers," IEEE Transactions on Microwave Theory and Techniques, vol. 59, pp. 2931 - 2942, Nov. 2011.

[4] N. Singhal, N. Nidhi, and S. Pamarti, "A power amplifier with minimal efficiency degradation under back-off," in IEEE International Symposium on Circuits and Systems, May 2010, pp. 1851-4.

[5] N. Singhal, N. Nidhi, R. Patel, and S. Pamarti, "A zero-voltage-switching contour-based power amplifier with minimal efficiency degradation under back-off," IEEE Transactions on Microwave Theory and Techniques, vol. 59, pp. 1589-1598, June 2011.

[6] D. Calvillo-Cortes, M. Acar, M. van der Heijden, M. Apostolidou, L. de Vreede, D. Leenaerts, and J. Sonsky, "A 65nm CMOS pulse-widthcontrolled driver with $8 \mathrm{Vpp}$ output voltage for switch-mode RF PAs up to $3.6 \mathrm{GHz}$," in IEEE International Solid-State Circuits Conference Digest of Technical Papers (ISSCC), Feb. 2011, pp. 58-60.

[7] M. Ozen, C. Andersson, M. Thorsell, K. Andersson, N. Rorsman, C. Fager, M. Acar, M. van der Heijden, and R. Jos, "High efficiency RF pulse width modulation with tunable load network class-E PA," in IEEE 12th Annual Wireless and Microwave Technology Conference (WAMICON), April 2011.

[8] C. M. Andersson, N. Ejebjörk, A. Henry, S. Andersson, E. Janzén, H. Zirath, and N. Rorsman, "A SiC varactor with large effective tuning range for microwave power applications," IEEE Electron Device Letters, vol. 32, pp. 788-790, June 2011.

[9] H. Cao, H. Nemati, A. S. Tehrani, T. Eriksson, J. Grahn, and C. Fager, "Linearization of efficiency-optimized dynamic load modulation transmitter architectures," IEEE Transactions on Microwave Theory and Techniques, vol. 58, April 2010.

[10] S. A. Doost, T. Eriksson, and C. Fager, "Digital predistortion using a vector switched model," IEEE Transactions on Microwave Theory and Techniques, vol. 60, no. 4, pp. 1166 -1174, April 2012. 